\title{
The Impact of High Intensity Interval Training On Lipid Profile, Inflammatory Markers and Anthropometric Parameters in Inactive Women
}

\begin{abstract}
Nasrin Zaer Ghodsi (MSc)
Department of Physical Education, Faculty of Physical Education and Sport Sciences, University of Urmia, Urmia, Iran

Mohammad Reza Zolfaghari (PhD)

Department of Exercise physiology, Faculty of Physical Education and Sport Sciences, University of Urmia, Urmia, Iran

Amir Fattah (MSc)

Department of Clinical Biochemistry, Department of Clinical Biochemistry, Faculty of Medicine, Tabriz University of Medical Sciences, Tabriz, Iran
\end{abstract}

Corresponding author: Mohammad Reza Zolfaghari

Email: zolfaghari60@gmail.com

Tell: +989143413941

Address: Assistant professor of Exercise physiology, Faculty of Physical Education and Sport Sciences, University of Urmia, Urmia, Iran

Received : 23 Aug 2014

Final edit: 30 Aug 2014

Accepted: 17 Sep 2014

\section{ABSTRACT}

Background and Objective: High-intensity interval training (HIIT) is a recently proposed exercise protocol, which is time-effective. The aim of this study was to evaluate the effect of HIIT for 8 weeks on the lipid profile, C-reactive protein (CRP), fasting blood sugar (FBS) and anthropometric parameters of young women who do not exercise.

Methods: In this study, 20 young physically inactive women performed HIIT workouts for 8 weeks and 3 sessions per week. The training protocol consisted of 10-times treadmill running for 15 seconds at maximum effort and then 30 seconds of resting. Blood samples were taken while fasting, a day before and after the training and then the considered parameters were measured. Wilcoxon test was used to compare the obtained data.

Results: HIIT significantly reduced FBS, cholesterol, low-density lipoprotein, highdensity lipoprotein / cholesterol ratio and CRP while increasing the HDL levels. There was a significant difference in the weight, body fat percentage, waist circumference, abdominal circumference and chest circumference of the subjects before and after the training ( $p$ $<0.05)$.

Conclusion: HIIT can improve lipid, inflammatory and anthropometric parameters, thus it can be considered as a suitable alternative to time-consuming exercises, especially for physically inactive women who like to spend less time to achieve optimal physical wellness and body fitness.

Keywords: High intensity interval training, anthropometric profile, exercise, lipids, Creactive protein. 


\section{INTRODUCTION}

Physical inactivity and subsequent weight gain cause problems such as high blood pressure, hypercholesterolemia and insulin resistance. The lack of sufficient time and time consuming exercise programs are among the main causes of reduced physical activity in various members of the society, especially women. Moreover, the majority of exercise protocols for reducing fat rely on long-term and regular exercises such as walking, which have little beneficial effects on weight loss and body fat burning (1). Therefore, various studies are seeking new exercise strategies that are time-effective with beneficial effects on individuals' health. High-intensity interval training (HIIT) is one of the recently proposed protocols, which consists several sessions of running at maximum speed on treadmill followed by short breaks, so that each session can take from 6 seconds to 4 minutes. This protocol is time-effective and therefore can replace traditional time-consuming protocols for women who do not have enough time to exercise. Many studies have been performed on the impact of HIIT on the physical health of obese, inactive and even athletes and demonstrated its benefits (2). Some studies considered the impact of HIIT on fat and body weight loss and acknowledged that this protocol increases fat oxidation, especially in post-training days. This increase was reported as $18 \%$ after two weeks by a study (3). Tremblay et al. reported that HIIT for 24 weeks can reduce subcutaneous and abdominal fat in young women more than continuous aerobic training (4). Whyte et al. also observed that HIIT for two weeks significantly reduces waist circumference of obese men (3). Creactive protein (CRP) is produced by the liver as an acute phase inflammatory response and is used as a risk marker for cardiovascular disease. Epidemiological studies have shown that high concentrations of CRP are significantly associated with heart attack, insulin resistance, obesity, high triglyceride and low high-density lipoprotein (HDL) level. The impact of HIIT on the level of serum CRP is not completely clear. Nevertheless, there is a possibility that HIIT can affect lipid profile in long-term and its improvement can reduce CRP as an inflammatory factor. Few studies have evaluated the effect of HIIT on blood glucose levels. It was proposed in a study that, HIT is more effective than other protocols for glycemic control in patients with type 2 diabetes (5). Given the direct link between insulin resistance and hyperglycemia with CRP level, lowered blood glucose level after HIIT may also play a role in CRP reduction. Thus, HIIT is likely to reduce the risk factors for diseases such as diabetes and atherosclerosis by improving lipid profile and reducing blood glucose level and subsequently CRP. This study aimed to evaluate the effect of 8-weeks HIIT on lipid profile, CRP and fasting blood glucose level of young women who do not exercise, in addition to determining the changes in anthropometric parameters of the subjects of this study.

\section{MATHERIAL AND METHODS}

In this semi-experimental study, 20 inactive women from the city of Bonab were participated with mean age of $24.9 \pm 4.3$ years. After giving the necessary information and knowledge about the study processes and objectives, informed written consent was obtained from all the participants. Subjects included individuals with no history of at least one year of regular exercise as well as those without cardiovascular disease, diabetes and inflammatory disorders such as rheumatoid arthritis, liver and kidney diseases. Before the start of the training, anthropometric characteristics including height, weight, body fat, body mass index (BMI), waist circumference, abdominal circumference, hip circumference, arm circumference, chest circumference and hip circumference were assessed. Caliper Harpenden protocol and three points skinfold measurement (Chest, Abdomen, Thigh) were used to measure body fat percentage. Twenty-four hours before the training, venous blood samples were taken from all subjects while fasting and serums were isolated by centrifugation. The samples were kept at $70{ }^{\circ} \mathrm{C}$ for the following necessary experiments. Then, HIIT workouts were performed on treadmill for 8 weeks and 3 sessions per week. Training protocol consisted of an initial warming for one minute at speed of $5 \mathrm{~km} / \mathrm{h}$ and then 15 seconds running at maximum effort (almost at the speed of $11 \mathrm{~km} /$ h) and 30 seconds of resting. This protocol was repeated 10 times per session. Finally, 48 hours after the last training session, fasting blood sampling was performed again, serums were separated and anthropometric parameters were measured. CRP, FBS, cholesterol, 
triglycerides, HDL and LDL of the serum samples taken before and after the trainings were measured by an autoanalyser (Hitachi 911, Japan) and using Pars Azmoon kits (Iran). Data Analysis: The collected data were analyzed using SPSS-19 statistical software. Nonparametric statistical tests were used due to small sample size. Wilcoxon test (paired Ttest for nonparametric data) was used for the comparison of quantitative parameters before and after the trainings. The Pearson correlation test was used to assess the possible correlation between two different parameters. Finally, $\alpha=0.05$ was considered as the statistical significance level.

\section{RESULTS}

The average of height, weight, BMI, body fat percentage, waist circumference, hip circumference, abdominal circumference, arm circumference, chest circumference and hip circumference of the subjects before and after the 8-weeks HIIT workouts are shown in Table 1. The results show that this training protocol significantly reduced the weight, body fat percentage, waist circumference, abdominal circumference and chest circumference of the subjects, while no significant change was observed in the rest of the anthropometric parameters. The findings also showed that this training notably changed the serum parameters of subjects by significantly reducing the fasting blood glucose level, cholesterol, LDL, HDL/cholesterol ratio and CRP level. Furthermore, it significantly increased serum HDL levels in the subjects of this study. No significant change was observed in the triglyceride levels after the training (Table 1).

Table 1- Mean ( \pm SD) of the anthropometric parameters, serum levels of fasting blood glucose, cholesterol, triglycerides, HDL, LDL and CRP of the participants before and after 8 weeks of HIIT

\begin{tabular}{|c|c|c|c|}
\hline P-Value & After the training & Before the training & Anthropometric parameters \\
\hline 1 & $160 / 2 \pm 6 / 12$ & $160 / 20 \pm 6 / 12$ & Height $(\mathrm{cm})$ \\
\hline $0 / 008$ & $75 / 9 \pm 6 / 28$ & $76 / 67 \pm 6 / 43$ & Weight (Kg) \\
\hline $0 / 812$ & $29 / 73 \pm 2 / 45$ & $29 / 86 \pm 2 / 48$ & BMI $\left(\mathrm{Kg} / \mathrm{m}^{2}\right)$ \\
\hline 0/007 & $36 / 81 \pm 3 / 8$ & $37 / 56 \pm 3 / 54$ & Body fat percentage \\
\hline $0 / 007$ & $89 / 4 \pm 7 / 3$ & $92 / 1 \pm 7 / 27$ & Waist circumference $(\mathrm{cm})$ \\
\hline 0/007 & $100 / 7 \pm 6 / 61$ & $104 / 4 \pm 6 / 67$ & Abdominal circumference $(\mathrm{cm})$ \\
\hline $0 / 063$ & $59 \pm 5 / 24$ & $59 / 6 \pm 5 / 39$ & Thigh circumference $(\mathrm{cm})$ \\
\hline 0/149 & $107 / 4 \pm 5 / 21$ & $108 / 7 \pm 5 / 2$ & Hip circumference $(\mathrm{cm})$ \\
\hline $0 / 083$ & $32 / 1 \pm 2 / 8$ & $32 / 4 \pm 3 / 02$ & Arm circumference (cm) \\
\hline 0/039 & $106 / 8 \pm 4 / 68$ & $107 / 6 \pm 4 / 59$ & Chest circumference $(\mathrm{cm})$ \\
\hline $0 / 022$ & $82 / 7 \pm 7 / 21$ & $90 / 4 \pm 8 / 84$ & Fasting blood glucose (mg/dl) \\
\hline $0 / 009$ & $173 / 3 \pm 19 / 06$ & $184 / 9 \pm 17 / 6$ & Cholesterol (mg/dl) \\
\hline $0 / 097$ & $143 / 9 \pm 74 / 03$ & $177 / 2 \pm 59 / 93$ & Triglyceride (mg/dl) \\
\hline $0 / 005$ & $52 / 6 \pm 6 / 27$ & $42 / 8 \pm 3 / 7$ & HDL (mg/dl) \\
\hline $0 / 03$ & $91 \pm 12 / 47$ & $96 / 5 \pm 14 / 96$ & LDL $(\mathrm{mg} / \mathrm{dl})$ \\
\hline $0 / 013$ & $4 / 05 \pm 0 / 46$ & $3 / 53 \pm 0 / 3$ & HDL/Cholesterol ratio \\
\hline $0 / 013$ & $6 / 68 \pm 3 / 35$ & $7 / 83 \pm 3 / 68$ & $\mathrm{CRP}(\mathrm{mg} / \mathrm{L})$ \\
\hline
\end{tabular}

\section{DISCUSSION}

The importance of exercise and physical activity is perfectly clear in the prevention of cardiovascular disease, diabetes and metabolic syndrome as well as weight control and body fitness. On the other hand, most popular exercise protocols are performed on a regular basis and in long-term and require a considerable amount of time. However, women in the society are unable to have regular physical activity due to the lack of sufficient time (1). Thus, new exercise protocols have been introduced that can have beneficial effects on human health and body fitness in a short time. HIIT is one of these protocols, which consists several short running sessions at maximum speed and then resting. Various studies have examined the efficacy of this method in healthy men and women (2). In this study, the effect of HIIT training for 8 weeks on the lipid profile, blood glucose level, inflammatory marker and anthropometric parameters of young inactive women were evaluated. The results showed the useful effects of these trainings even for a short time. Our study revealed that this short-term training can improve many anthropometric parameters of women, as the waist, chest and abdominal circumference, weight and body fat percentage of these individuals decreased significantly. Reduction of waist circumference was reported in a similar study for two weeks in 
obese men, but other parameters did not differ (3). This may be due to the low duration of trainings in the mentioned study, since anthropometric changes require more time. The lack of significant differences in other anthropometric parameters in the present study may also be due to the short duration of the trainings. In similar studies with over 12 weeks of training, body weight and subcutaneous and abdominal fat were significantly decreased (4). Trapp et al., after evaluating the effects of 15 -weeks HIIT workouts on young women found that the body weight and subcutaneous and abdominal fat were reduced by $2 \%, 10 \%$ and $10 \%$, respectively. However, the exercise protocol in the mentioned study consisted 8 seconds of pedaling at maximum effort and 12 seconds of resting, with 60 repeats per session (6). The mechanisms involved in weight loss and body fat mass reduction following the HIIT are not exactly clear. However, it may be due to the increased fat oxidation during HIIT and subsequent days, since it is demonstrated that the level of enzymes and hormones involved in lipolysis are increased. Increase of hormones such as catecholamine, cortisol and growth hormone has been observed during HIIT. It is likely that these hormones, particularly catecholamine that have betaadrenergic receptors in adipose tissue may induce lipolysis and contribute to increased fat oxidation. The increased activity of $\beta$-hydroxy coenzyme A dehydrogenase, which is the key enzyme in regulating fat oxidation, is also demonstrated (4). Another possible mechanism of decreased appetite after the training may be due to the production of corticotropin-releasing factor (CRF), which is a peptide with anorexic actions (7). The findings revealed that a period of 8-weeks HIIT can improve the lipid profile leading to a significant reduction of cholesterol and LDL and increased HDL. It is obvious that reduced LDL and cholesterol along with increased HDL can reduce the risk of cardiovascular diseases. It is found that high-intensity training can reduce serum cholesterol levels, an effect that is not observed in low intensity trainings (8). Therefore, as demonstrated by the present study, HIIT workouts can remarkably reduce cholesterol levels. In agreement with the findings of this study, it has been reported that high-intensity training significantly decreases HDL / cholesterol ratio, which is a more suitable marker for predicting the risk of coronary artery disease (9). Studies have shown that acute and chronic exercises reduce plasma triglycerides, an effect which was not observed in the present study. Exercise lowers triglycerides as well as eliminating very low density lipoprotein (VLDL) which ultimately leads to decreased triglyceride content of plasma and the liver. However, since VLDL was not measured in this study, no correct interpretation can be provided for the effects of HIIT on the triglyceride levels. Fasting blood glucose significantly reduced in the subjects after the training, which represents the beneficial effect of HIIT in glycemic control of these individuals. Thus, HIIT can be highly effective in preventing disorders such as diabetes. Increased insulin sensitivity may be a possible cause of decreased blood glucose, as Whyte et al. have shown that insulin sensitivity increases after HIIT and identified the positive correlation of training intensity with level of insulin sensitivity increase. Despite the short time of training ( 8 weeks), the reduce blood glucose level observed in the present study can be attributed to the type of exercises that were done with high intensity. Due to increased metabolic stress, CRP that is a risk marker for cardiovascular diseases increases during acute and long exercises with high intensity such as marathon. It has been reported that increased CRP level in such situations may be due to production of stress hormones, which eventually causes inflammatory response by affecting the immune system. Increase of inflammatory mediators and cytokines such as Interleukin1,6 and granulocyte colony-stimulating factor as well as changes in the pattern of neutrophils' gene expression have been reported in these situations (10). However, the present study showed that HIIT not only does not increase CRP but decreases serum CRP levels, which indicate the anti-inflammatory and protective effects of this type of training. High amount of lipids can increase blood glucose by causing insulin resistance, thus HIIT can reduce blood glucose level in longterm by improving the lipid status. Moreover, HIIT can reduce these parameters by increasing the oxidation of sugars and lipids. On the other hand, previous studies have reported that decreased lipid and glucose levels can reduce CRP. Improvement of the 
parameters involved in cardiovascular disease and diabetes on one hand and reduced BMI and anthropometric factors on the other, can improve health status and prevent metabolic and cardiovascular diseases in women who lack regular exercise.

\section{CONCLUSION}

HIIT for 8 weeks improves the lipid profile, blood glucose and serum CRP levels of physically inactive women. This type of training in a short time can also reduce the anthropometric parameters such as waist circumference, chest circumference, abdominal circumference, weight and fat percentage, which are important in terms of

\section{REFERENCES}

1. Wu T, Gao X, Chen M, Van Dam R. Long-term effectiveness of diet-plus-exercise interventions vs. dietonly interventions for weight loss: a meta-analysis. Obesity Reviews. 2009;10(3):313-23.

2. Whyte LJ, Ferguson C, Wilson J, Scott RA, Gill JM. Effects of single bout of very high-intensity exercise on metabolic health biomarkers in overweight/obese sedentary men. Metabolism. 2013;62(2):212-9.

3. Whyte LJ, Gill JM, Cathcart AJ. Effect of 2 weeks of sprint interval training on health-related outcomes in sedentary overweight/obese men. Metabolism. 2010; 59(10): 1421-8.

4. Limbaugh JD, Wimer GS, Long LH, Baird WH. Body fatness, body core temperature, and heat loss during moderate-intensity exercise. Aviation, space, and environmental medicine. 2013; 84(11): 1153-8.

5. Gillen JB, Little JP, Punthakee Z, Tarnopolsky M, Riddell M, Gibala M. Acute high intensity interval exercise reduces the postprandial glucose response and prevalence of hyperglycaemia in patients with type 2 diabetes. Diabetes, Obesity and Metabolism. 2012;14(6):575-7. doi: 10.1111/j.14631326.2012.01564.x. physical health and fitness of women. Although this study lacked a control group to determine the effect of HIIT workouts in comparison with the traditional exercises, it can be concluded that HIIT can be a suitable alternative to traditional exercises due to its beneficial effects and time-effectiveness.

\section{ACKNOWLEDGMENTS}

The authors would like to thank the colleagues in the Department of Physiology, Faculty of Physical Education, University of Urmia and all the participants in the study for their assistance and cooperation.

\section{CONFLICT OF INTEREST}

Therer are no conflicts of interest.

6. Trapp E, Chisholm D, Freund J, Boutcher S. The effects of high-intensity intermittent exercise training on fat loss and fasting insulin levels of young women. International journal of obesity. 2008; 32(4): 684-91.

7. Bilski J, Teległów A, Zahradnik-Bilska J, Dembiński A, Warzecha Z. Effects of exercise on appetite and food intake regulation. Medicina Sportiva. 2009;13(2):82-94.

8. Heydari M, Boutcher YN, Boutcher SH. The effects of high-intensity intermittent exercise training on cardiovascular response to mental and physical challenge. International Journal of Psychophysiology. 2013;87(2):141-6. doi: 10.1016/j.ijpsycho.2012.11.013.

9. De Groot P, Hjeltnes N, Heijboer A, Stal W, Birkeland K. Effect of training intensity on physical capacity, lipid profile and insulin sensitivity in early rehabilitation of spinal cord injured individuals. Spinal cord. 2003;41(12):673-9.

10. Peake JM, Suzuki K, Wilson G, Hordern M, Nosaka $\mathrm{K}$, Mackinnon L, et al. Exercise-induced muscle damage, plasma cytokines, and markers of neutrophil activation. Med Sci Sports Exerc. 2005;37(5):737-45. 